\title{
Pathways related to mitochondrial dysfunction in cartilage of endemic osteoarthritis patients in China
}

\author{
LI ChunYan ${ }^{1,2}$, WANG WeiZhuo ${ }^{3}$, GUO Xiong ${ }^{1 *}$, ZHANG Feng ${ }^{1}$, MA WeiJuan ${ }^{1}$, \\ ZHANG YinGang ${ }^{4}$, LI YouFen ${ }^{5}$, BAI YiDong ${ }^{6}$ \& LAMMI Mikko J \\ ${ }^{1}$ Faculty of Public Health, Medical College of Xi'an Jiaotong University, Key Laboratory of Environment and Gene Related Diseases of Min- \\ istry of Education, Key Laboratory of Trace elements and Endemic Diseases of Ministry of Health, Xi'an 710061, China; \\ ${ }^{2}$ Institute of Health Education of Xi'an, Xi'an 710004, China; \\ ${ }^{3}$ Department of Orthopedics Surgery, Second Hospital, Xi'an Jiaotong University, Xi'an 710004, China; \\ ${ }^{4}$ Department of Orthopedics Surgery, First Hospital, Xi'an Jiaotong University, Xi'an 710061, China; \\ ${ }^{5}$ School of Life Science and Technology, Xi'an Jiaotong University, Xi'an 710049, China; \\ ${ }^{6}$ Department of Cellular and Structural Biology, University of Texas Health Sciences Center at San Antonio, San Antonio, Texas 78229, USA; \\ ${ }^{7}$ Department of Anatomy, Institute of Biomedicine, University of Kuopio, Kuopio, FIN-70211, Finland
}

Received June 19, 2012; accepted October 17, 2012

\begin{abstract}
In this paper, we present the first evidence of differences in the mitochondria-related gene expression profiles of adult articular cartilage derived from patients with Kashin-Beck disease and normal controls. The expression of 705 mitochondria-related genes was analyzed by mitochondria-related gene expression analysis and ingenuity pathways analysis. Mitochondria-related gene expression analysis identified 9 up-regulated genes in Kashin-Beck disease based on their average expression ratio. Three canonical pathways involved in oxidative phosphorylation, apoptosis signaling and pyruvate metabolism were identified, which indicate the involvement of mitochondrial dysfunction in the pathogenesis of Kashin-Beck disease.
\end{abstract}

Kashin-Beck disease, microarray, mitochondrial dysfunction, IPA analysis

Citation: $\quad$ Li C Y, Wang W Z, Guo X, et al. Pathways related to mitochondrial dysfunction in cartilage of endemic osteoarthritis patients in China. Sci China Life Sci, 2012, 55: 1057-1063, doi: 10.1007/s11427-012-4418-4

Kashin-Beck disease (KBD) is a chronic, endemic deforming osteoarthrosis, characterized by chondrocyte necrosis and apoptosis, cartilage degeneration and matrix degradation [1]. KBD mainly affects older children, damaging the hyaline cartilage during development and becoming symptomatic in adults who exhibit short stature, joint deformities, and other features caused by impaired epiphyseal growth and ossification. The main characteristic pathological changes of KBD are chondrocyte necrosis, excessive apoptosis, and dedifferentiation in the deep zone of cartilage (Figure 1). Currently, KBD is mainly prevalent in China,

*Corresponding author (email: guox@mail.xjtu.edu.cn)
Siberia, and North Korea [2,3]. It has been reported that more than 2.5 million people suffer from KBD, and about 30 million people are at risk of KBD in China [4]. KBD not only significantly reduces patients' quality of life, but also brings heavy medical and financial burdens to local governments.

The etiology of KBD remains unclear. Various environmental etiologic hypotheses have been proposed, such as selenium deficiency, serious cereal contamination by mycotoxin-producing fungi, and high humic acid levels in drinking water [5]. However, none of these hypotheses can completely explain the pathological changes seen in KBD. Recently, several studies were conducted to investigate the 


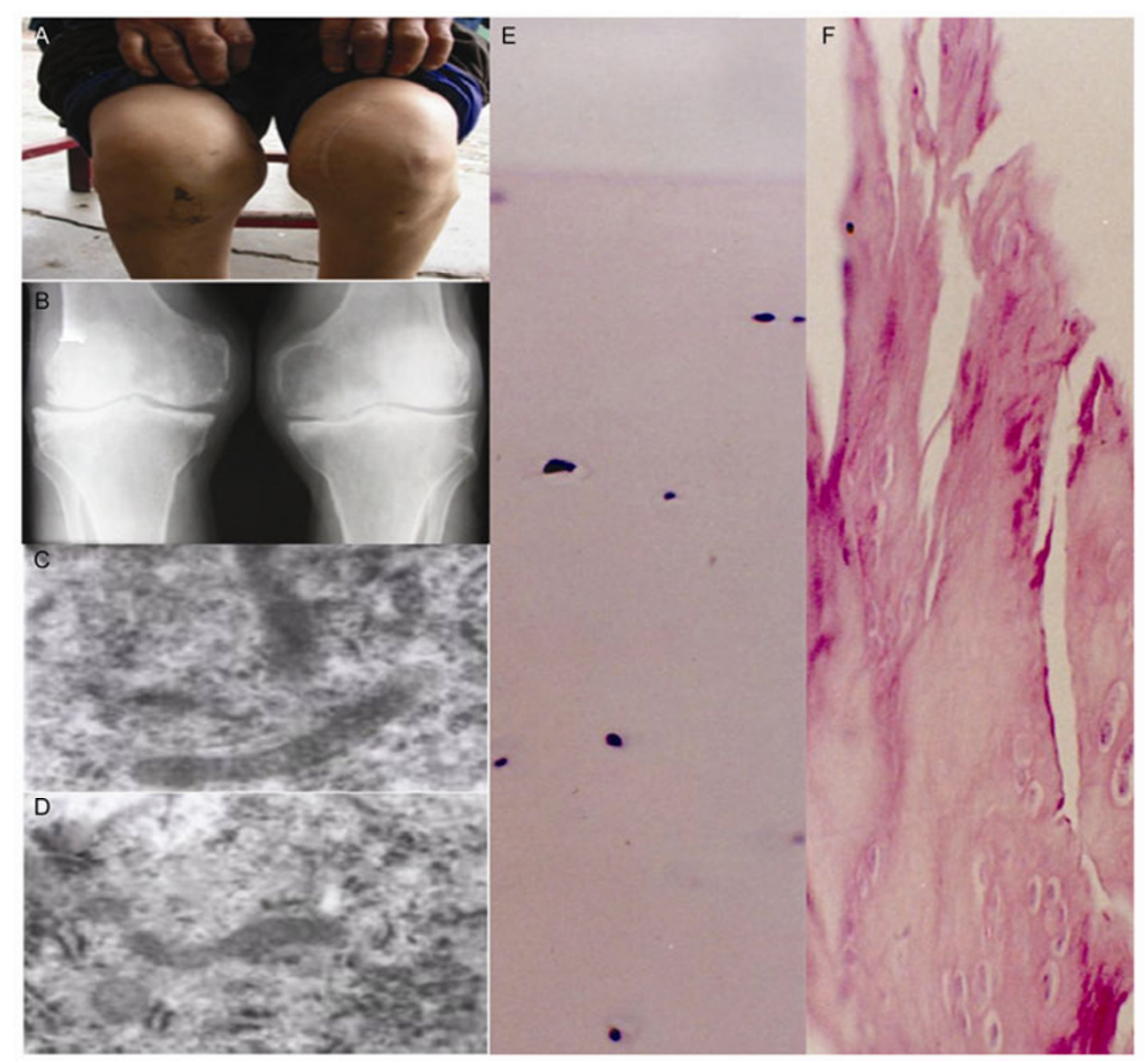

Figure 1 The characteristics of Kashin-Beck disease (KBD). The characteristic appearance and radiographic images of knees in KBD (grade III, male, 47 years old, A and B). The damage of mitochondrion in KBD chondrocyte is shown by the electron microscope (C, mitochondrion in the culture chondrocyte of KBD; D, the normal mitochondrion; 20000). Compared with healthy control tissue (E), KBD articular cartilage (F) shows an eroded surface and chondronecrosis (E and F, H.E. $20 \times 100)$.

roles of mitochondria and apoptosis in the pathogenesis of KBD. The findings showed that abnormal mitochondrial function and cell death were involved in the pathophysiology of this disease [6,7], suggesting that mitochondrial dysfunction and apoptosis play important roles in the pathogenesis of KBD. However the detailed mechanisms involved in the mitochondrial dysfunction and apoptosis underlying the cartilage lesions in KBD remained unclear.

Ingenuity pathways analysis (IPA) is a widely used software tool [8-11] which can help researchers to model, analyze, and understand the complex biological and chemical systems at the core of life-science research. In our study, IPA helped to investigate the biology of KBD at multiple levels by integrating data from a variety of experimental platforms, providing insight into the molecular and chemical interactions, cellular phenotypes, and disease processes of KBD.

To investigate the potential roles of mitochondrial dysfunction in the pathogenesis of $\mathrm{KBD}$, we compared the mitochondria-related gene expression profiles of cartilage derived from KBD patients and normal controls. Both mitochondria-related gene expression analysis and IPA were conducted to identify the key genes and pathways contrib- uting to the pathogenesis of KBD.

\section{Materials and methods}

All studies were approved by the Human Ethics Committee, Xi'an Jiaotong University. All patients, or relatives of donors, provided informed consent.

\subsection{Cartilage sample collection}

Specimens of human articular cartilage were collected from 9 KBD adults and 9 normal controls matched for age and sex (Table 1). KBD patients were diagnosed as having second and third degree KBD according to the diagnosing criteria of KBD in China (diagnostic code GB16395-1996). The KBD patients came from the KBD prevalent areas of Linyou and Yongshou in the Shaanxi Province of China. Normal human knee cartilage samples were collected from the knees of fresh cadavers within $8 \mathrm{~h}$ of death caused by traffic accidents. All normal control samples were from non-KBD areas. The KBD status of normal controls was diagnosed by histological examination with H\&E staining, 
Table 1 KBD-control pairs used for microarray and quantitative RT-PCR analysis

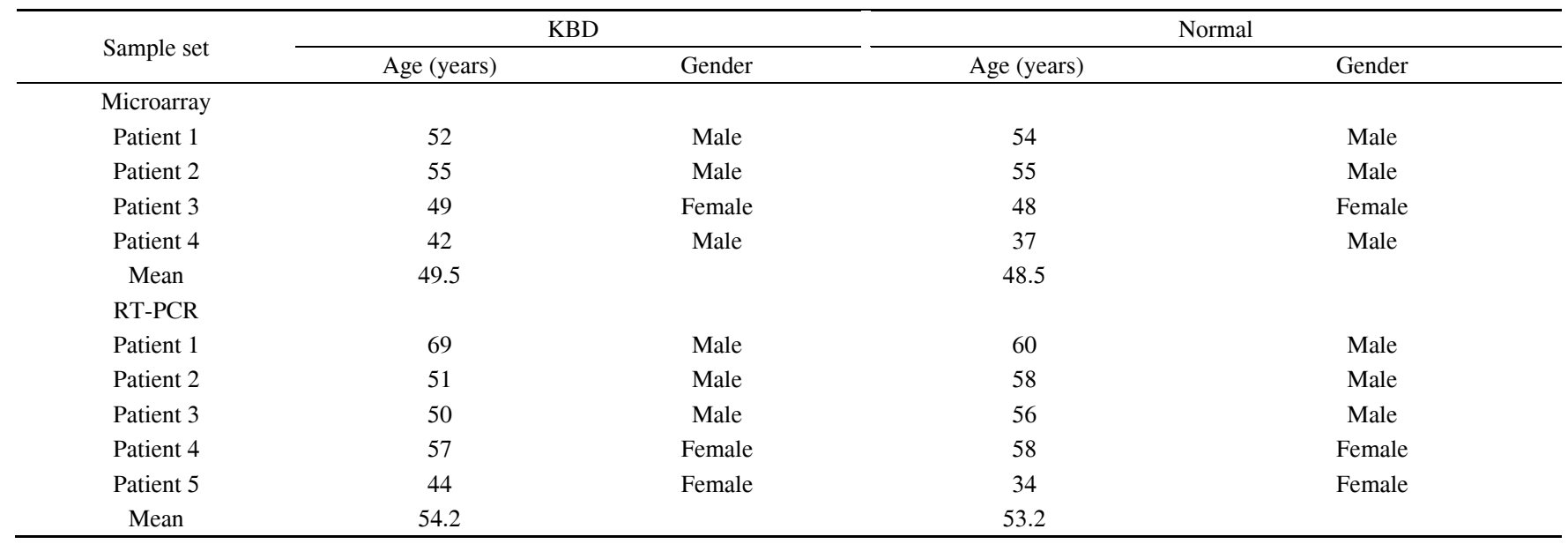

and subjects with osteoarthritis (OA), rheumatoid arthritis and genetic bone and cartilage diseases were excluded. All cartilage samples were derived from the same anatomic area of the femoral condyle of the knee. Cartilage tissue from the femoral condyles was dissected and rapidly frozen in liquid nitrogen, then stored at $-80^{\circ} \mathrm{C}$ until used for RNA isolation. Four pairs of KBD and control samples were subjected to microarray analysis, and the others were used for quantitative real-time reverse transcription polymerase chain reaction (RT-PCR) (Table 1).

\subsection{RNA preparation}

Frozen cartilage from each sample was first rapidly ground in liquid nitrogen using a freezer mill (SPEX CertiPrep, Metuchen, NJ). Total RNA was then isolated from cartilage samples using the Agilent Total RNA Isolation Mini kit (Agilent Technologies, Santa Clara, CA) following the manufacturer's recommended protocol. The quality and integrity of extracted total RNA were evaluated by $1 \%$ agarose gel electrophoresis.

\subsection{Microarray hybridization}

The isolated total RNA was first transcribed into complementary DNA (cDNA), and labeled with CyDye using the Amino Allyl MessageAmp aRNA Kit (Applied Biosystems, Austin, TX). For each KBD-control pair, $0.5 \mu \mathrm{g}$ of labeled cRNA was purified separately and mixed together with hybridization buffer (Agilent in Situ Hybridization Plus kit). An Agilent Human 1A 22k Whole Genome microarray (G4110B), containing 22575 oligonucleotides probes representing 21073 human genes, was then applied for microarray hybridization following the Agilent recommended protocol. Hybridization signals were recorded by an Agilent G52565BA scanner, and analyzed by Feature Extraction 9.3 (Agilent Technologies) and Spotfire 8.0 (Spotfire Inc., Cambridge, MA) software. The quality of fluorescent spots was evaluated by Feature Extraction 9.3. The fluorescent spots that failed to pass the quality control procedure were excluded from further analysis. Linear and LOWESS normalization implemented by Feature Extraction 9.3 were also conducted to eliminate possible dye-related bias in our microarray data. The gene expression data obtained from Spotfire 8.0 were imported into Excel spreadsheets (Microsoft Corp., Redmond, WA) for downstream data analysis.

\subsection{Mitochondria-related gene expression analysis}

To identify differentially-expressed genes, expression ratios were calculated for each gene. Genes with expression ratios $\leqslant 0.5$ or $\geqslant 2.0$ were regarded as significant in this study. Statistical $P$-values were also calculated for each gene using the Agilent $P$-value log ratio algorithm (Agilent Technologies), defined by

$$
P=1-\operatorname{Erf}\left(\frac{|x d e v|}{\sqrt{2}}\right)=\operatorname{Erfc}\left(\frac{|x d e v|}{\sqrt{2}}\right),
$$

in which $\operatorname{Erf}(x)$ is calculated as

$$
\operatorname{Erf}(x)=\frac{2}{\sqrt{P i}} \int_{0}^{x} \mathrm{e}^{-t^{2}} \mathrm{~d} t .
$$

$\operatorname{Erf}(x)$ represents twice the integral of the Gaussian distribution, with a mean value of 0 and variance of 0.5 , and $x d e v$ is the deviation of the $\log$ ratio from 0 . This calculation gives the statistical significance of the log ratio for each feature (i.e., transcript levels) between the red and green channels. Only $P$-values less than 0.05 were regarded as significant. Once significant genes were identified, we used the NCBI database to identify the relationship of each gene to cartilage or other possible contributory factors.

\subsection{Ingenuity pathways analysis}

IPA uses a Fisher's exact test to determine which biofunctions and canonical pathways are significantly linked to 
the genes of interest compared to the whole Ingenuity knowledge base. In this study we used IPA to identify mitochondria-related pathways involved in the pathophysiology of KBD. Briefly, web-based Ingenuity Pathway software (Ingenuity® Systems, www.ingenuity.com) was employed to examine the function and pathway of the different genes. Data sets containing gene identifiers and corresponding expression values were uploaded into Ingenuity Pathway software. Each gene identifier was mapped to its corresponding gene object in the Ingenuity Pathways Knowledge Base. Genes differentially expressed with $P<0.01$ were overlaid onto global molecular networks developed from information contained in the knowledge base. Canonical pathway analysis identified function-specific genes significantly present within the networks.

\subsection{Quantitative RT-PCR validation}

For quantitative RT-PCR, total RNA was prepared in the same way as for the oligonucleotide microarray analysis. These RNA samples were converted into cDNA using Superscript II reverse transcriptase (Invitrogen, Carlsbad, CA) and random primers. Quantitative RT-PCR was performed using the ABI 7500 Real-Time PCR system (Applied Biosystems, Foster City, CA) according to the manufacturer's instructions. Six genes with expression ratios $>2.0$ or $<0.5$ in our microarray analysis were selected for quantitative RT-PCR, including CASP8AP2 (NM_012115), BMF (NM_ 001003940), PAPSS2 (NM_001015880), POSTN (NM_ 006475), TACC1 (NM_006283) and TMSL8 (NM_021992). Glyceraldehyde-3-phosphate dehydrogenase (GAPDH) was simultaneously assayed by quantitative RT-PCR as an endogenous invariant control for data normalization. All primer and probe sets used for quantitative RT-PCR were supplied by TaqMan Gene Expression Assays (Applied Biosystems). The expression values of the 6 selected genes were normalized to the GAPDH values, and then used to calculate expression ratios. A paired t-test was conducted to assess the significance of differences in expression of the 6 genes between KBD and normal controls by quantitative RT-PCR.

\section{Results}

\subsection{Mitochondria-related gene expression analysis}

The 705 mitochondria-related genes were identified as mitochondria-related genes according to hMitChip 3.0 [12]. The expression level of these genes, which is from the whole human genome transcripts, was assessed in this study. SGEA results are presented in Table 2. We identified 9 genes with an average ratio up-regulated in KBD compared to normal controls. The 9 identified genes belong to various functional categories, mainly including apoptosis (3 genes), metabolism (3 genes), cytoskeleton and cell movement (1 gene) and signal transduction (1 gene).

\subsection{Ingenuity pathways analysis}

IPA analysis revealed 5 top bio-functions involving genes with altered expression in KBD cartilage compared to healthy cartilage. As shown in Table 3, the top 5 biofunctions were involved in the processes of cell death, cell morphology, DNA replication, recombination, and repair as well as post-translational modification. The function of cell death included the largest proportion of molecules.

Based on the biological functions of the identified pathways, we identified 4 interesting canonical pathways that may contribute to the pathogenesis of KBD (Table 4, Figure 2). The most significant canonical pathway identified by IPA is mitochondrial dysfunction with the highest ratio (the proportion of mitochondria-related genes in the pathway) in this study. The other 3 canonical pathways identified were involved in oxidative phosphorylation, apoptosis signaling and pyruvate metabolism.

\subsection{Quantitative RT-PCR analysis}

The quantitative RT-PCR analyses yielded results that were consistent with the microarray data, for the mRNA expression levels of CASP8AP2, BMF, PAPSS2, POSTN, TACC1 and TMSL8 (Figure 3). Specifically, expression levels of CASP8AP2, TMSL8 and PAPSS2 were higher

Table 2 List of mitochondria related genes up-regulated in KBD cartilage

\begin{tabular}{|c|c|c|c|c|}
\hline Gene name & Symbol & Public ID & Fold change ${ }^{a)}$ & $P$-value \\
\hline BCL2-antagonist/killer 1 & BAK1 & NM_001188 & $5.97 \pm 1.11$ & 0.01 \\
\hline Apoptotic protease activating factor & APAF1 & NM_181861 & $5.70 \pm 2.62$ & 0.01 \\
\hline Actin, alpha 2, smooth muscle, aorta & ACTA2 & NM_001613 & $4.03 \pm 3.14$ & 0.04 \\
\hline DEAD(Asp-Glu-Ala-Asp)box polypeptide 3,Y-linked & DDX3Y & NM_004660 & $5.97 \pm 4.81$ & $<10^{-3}$ \\
\hline Peptidylprolyl isomerase C (cyclophilin C) & PPIC & NM_000943 & $4.23 \pm 3.31$ & 0.04 \\
\hline Caspase 6, apoptosis-related cysteine protease & CASP6 & NM_001226 & $8.33 \pm 3.67$ & $<10^{-3}$ \\
\hline Lactate dehydrogenase A & LDHA & NM_005566 & $5.31 \pm 3.38$ & 0.02 \\
\hline Isocitrate dehydrogenase $2\left(\mathrm{NADP}^{+}\right)$, mitochondrial & $\mathrm{IDH} 2$ & NM_002168 & $4.31 \pm 0.53$ & 0.04 \\
\hline
\end{tabular}

a) The mean and SEM of the fold-change in expression of each gene. 


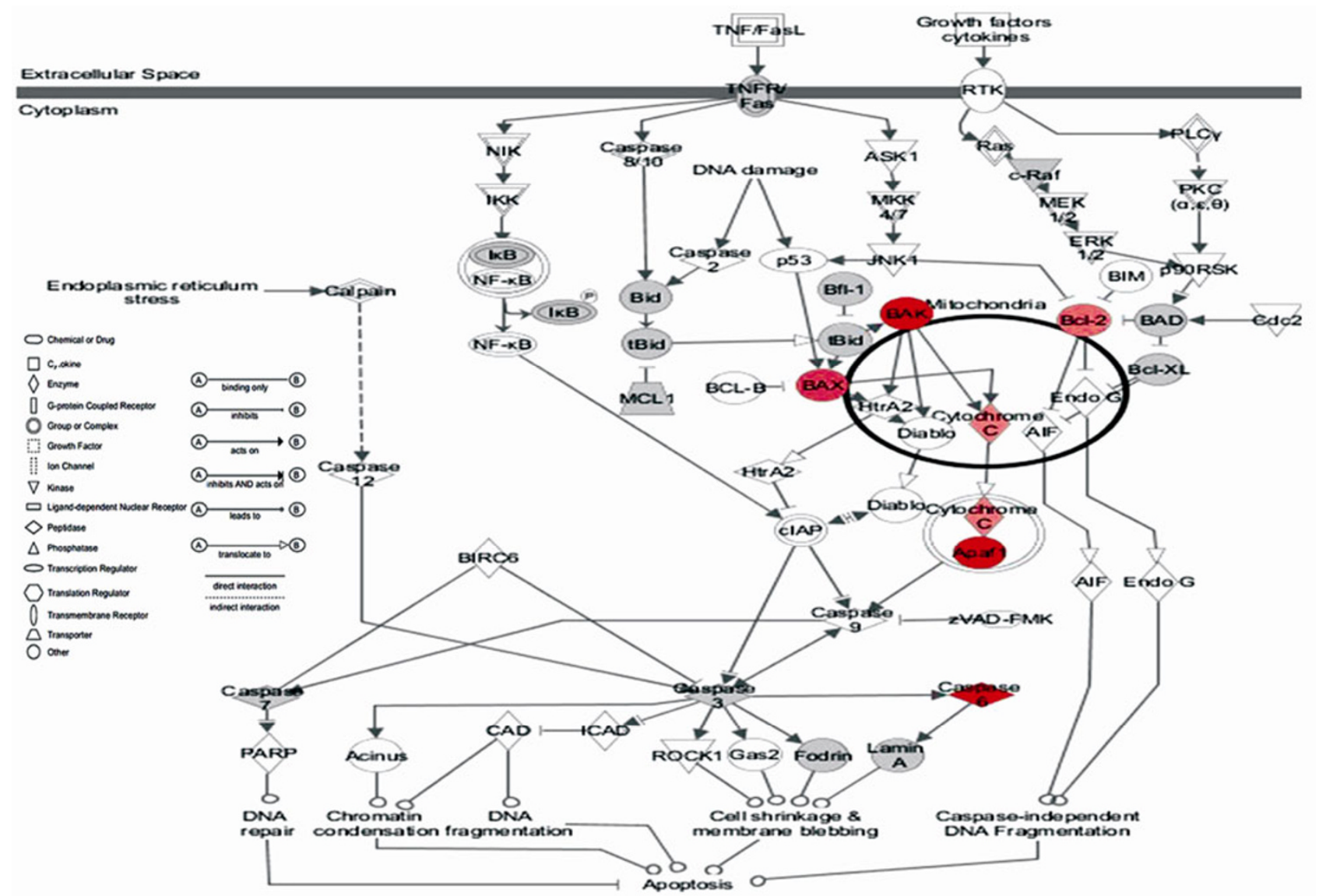

Figure 2 The apoptosis signaling pathway. Diagram showing the signaling pathways involved in the process of apoptosis, red indicates mitochondria-related genes which are included in the pathway.

Table 3 Top bio-functions of mitochondria-related genes identified in KBD cartilage vs. healthy cartilage ${ }^{\text {a) }}$

\begin{tabular}{ccc}
\hline Name & Molecules & $P$-value \\
\hline Cell death & 33 & $1.08 \times 10^{-6}$ \\
Cellular assembly and organization & 16 & $7.04 \times 10^{-6}$ \\
Cell morphology & 16 & $2.02 \times 10^{-7}$ \\
DNA replication, recombination, and repair & 14 & $5.14 \times 10^{-6}$ \\
Post-translational modification & 6 & $5.10 \times 10^{-7}$ \\
\hline
\end{tabular}

a) Molecules and $P$-value were calculated by IPA.

Table 4 Top canonical pathways of mitochondria-related genes identified in KBD cartilage vs. healthy cartilage ${ }^{\text {a) }}$

\begin{tabular}{cccc}
\hline Pathway name & Gene & Ratio \\
\hline Mitochondrial dysfunction & $1.49 \times 10^{-15}$ & 15 & 8.7 \\
Oxidative phosphorylation & $5.46 \times 10^{-8}$ & 9 & 6.1 \\
Apoptosis signaling & $5.68 \times 10^{-6}$ & 6 & 6.4 \\
Pyruvate metabolism & $3.50 \times 10^{-6}$ & 6 & 4.6 \\
\hline
\end{tabular}

a) The ratio and $P$-value were calculated by IPA. The ratio denotes the proportion of mitochondria-related genes in the pathway.

whereas those of TACC1, POSTN, and BMF were lower in KBD cartilage samples than in normal cartilage samples.

\section{Discussion}

In this paper, we present the first evidence showing that canonical pathways involved in oxidative phosphorylation, apoptosis signaling and pyruvate metabolism are related to the mitochondrial dysfunction observed in $\mathrm{KBD}$, as well as evidence for the underlying mechanism of mitochondrial dysfunction in the cartilage of patients with KBD which was previously not clear-cut [6]. 


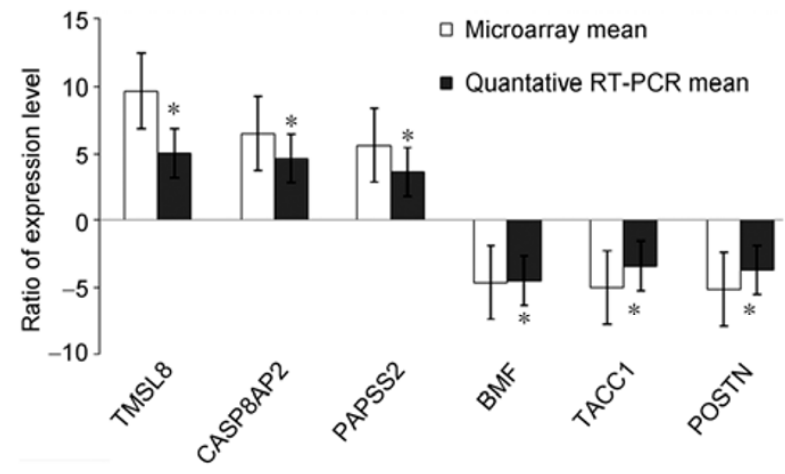

Figure 3 The results of quantitative RT-PCR. Histogram showing the expression ratio of the 6 selected genes, as measured by microarray (white bars, $n=4$ ) and quantitative RT-PCR (black bars, $n=5$ ). * indicates $P<0.05$, calculated by Student's paired $t$-test.

It has been well documented that mitochondria play a critical role in apoptosis [13]. In our study, apoptosis-related genes and their pathways (Table 4, Figure 2) were significantly up-regulated in KBD, and 3 of the 9 differentially-expressed genes identified were apoptosis-related, including BAK1, APAF1 and CASP6. Upregulation of these genes in KBD may result in excessive chondrocyte apoptosis and abnormal expression of the apoptosis-related genes BCL-2, BAX, FAS and iNOS in articular cartilage [5]. BAK1 is also known as BAK in NCBI. BAK regulates the intrinsic apoptosis pathway by promoting apoptosis and binding to anti-apoptotic family members including BCL-2 and BCL-XL [14,15].

CASP6 encodes a protein which is a member of the cysteine-aspartic acid protease (caspase) family. Sequential activation of caspases plays a central role in the execution-phase of cell apoptosis. APAF1 is related to caspase 9 and commits a cell to apoptosis [16,17]. Combining this evidence with that from our previous study [7] shed light on the role of mitochondria-related genes and their contribution to the mitochondria-mediated chondrocyte apoptosis underlying the pathogenesis of KBD.

An interesting finding in this study is that mitochondria-related genes and pathways were dependent on oxidative damage and dysfunctions of energy metabolism involved in the destruction of KBD chondrocytes, such as IDH2, 'oxidative phosphorylation' and 'pyruvate metabolism'. A marked increase in expression of IDH2 in KBD cartilage was observed compared with healthy cartilage. IDH2 plays a role in the metabolism of glutathione, which composes the important glutathione/glutathione disulfide anti-oxidant protecting system of the human body [18]. As a major component of the glutathione/glutathione disulfide anti-oxidant protection system, glutathione peroxidase 1 (GSH-Px1) is known to be associated with the risk of KBD [19]. The protective effect of GSH-Px1 largely depends on the presence of selenium, while low selenium is an important environmental risk factor for KBD. Therefore, the glutathione/glutathione disulfide anti-oxidant protection system plays a role in the pathogenesis of KBD.

Transport of pyruvate into the mitochondria is necessary for the oxidative decarboxylation of pyruvate to acetyl-CoA, and is fundamental to glucose oxidation and ATP production [20]. The oxidative phosphorylation (OXPHOS) system is composed of more than 85 proteins organized into five multisubunit, enzymatic protein complexes (I-V) and two electron carriers (cytochrome c, coenzyme Q). Complexes I-IV comprise the electron transport chain, which generates an electrochemical proton gradient across the mitochondrial inner membrane $(\Delta \psi \mathrm{m})$ [21]. KBD chondrocytes have been shown to have a lower $\Delta \psi \mathrm{m}$ : green fluorescence ratio than normal chondrocytes $(0.75,95 \%$ CI $0.71-0.79$ vs. 9.7, 95\% CI 6.71-12.69, $P \leqslant 0.0001)$ by flow cytometry [6].

The most significant canonical pathways in mitochondrial dysfunction, which are those involved in alterations in mitochondrial function and in mitochondrial damage, have been linked to a variety of cellular and organismal responses including apoptosis, neuromuscular disease, tumor pathogenesis, and aging [13,22-24]. Dysfunctions in complexes II, III and IV of the OXPHOS system have been found in KBD chondrocytes [6].

It is well known that mitochondria are important organelles contributing to energy metabolism, reactive oxygen species (ROS) production and apoptosis in the human body [25]. Mitochondria can produce ROS through the action of the mitochondrial electron transport chain (ETC), while ROS can lead to mitochondrial DNA (mtDNA) mutagenesis [26]. MtDNA damage and mutations lead to progressive respiratory chain dysfunction and increasing ROS production as a consequence of mitochondrial dysfunction [27,28]. Mitochondrial dysfunction and subsequent abnormal energy metabolism and apoptosis might play an important role in the pathogenesis of KBD.

In a summary, three canonical pathways involved in oxidative phosphorylation, apoptosis signaling and pyruvate metabolism were identified by the mitochondria-related gene expression profiles of KBD cartilage, which related to mitochondrial dysfunction in the pathogenesis of KBD. These results may help to elucidate the differences and similarities of the molecular mechanism underlying the development of cartilage damage in KBD and other osteoarthroses.

We thank the orthopedic surgeons and nursing staff of the Department of Orthopedics or Trauma in the Xi'an Red Cross Hospital, the Second Affiliated Hospital of Xi'an Jiaotong University and Shaanxi Endemic Disease Hospital for support and cooperation in the collection of cartilage specimens. This work was supported in part by the National Natural Science Foundation of China (Grant No. 30972556), the Key Scientific and Technological Innovation Special Projects of Shaanxi "13115” of China (Grant No. 2009ZDKG-79) and the Specialized Research Fund for the Doctoral Program of Higher Education of China (Grant No. 20090201110049). 
1 Duan C, Guo X, Zhang X D, et al. Comparative analysis of gene expression profiles between primary knee osteoarthritis and an osteoarthritis endemic to northwestern china, kashin-beck disease. Arthritis Rheum, 2010, 62: 771-780

2 Downey $\mathrm{C} \mathrm{M}$, Horton $\mathrm{C} \mathrm{R}$, Carlson $\mathrm{B} \mathrm{A}$, et al. Osteochondroprogenitor-specific deletion of the selenocysteine trna gene, trsp, leads to chondronecrosis and abnormal skeletal development: A putative model for kashin-beck disease. PLoS Genet, 2009, 5: e1000616

3 Moreno-Reyes R, Suetens C, Mathieu F, et al. Kashin-beck osteoarthropathy in rural tibet in relation to selenium and iodine status. N Engl J Med, 1998, 339: 1112-1120

4 Guo X, Zuo H, Cao C X, et al. Abnormal expression of col x, pthrp, tgf-beta, bfgf, and vegf in cartilage with kashin-beck disease. J Bone Miner Metab, 2006, 24: 319-328

5 Wang S J, Guo X, Zuo $\mathrm{H}$, et al. Chondrocyte apoptosis and expression of bcl-2, bax, fas, and inos in articular cartilage in patients with kashin-beck disease. J Rheumatol, 2006, 33: 615-619

6 Liu J T, Guo X, Ma W J, et al. Mitochondrial function is altered in articular chondrocytes of an endemic osteoarthritis, kashin-beck disease. Osteoarthritis Cartilage, 2010, 18: 1218-1226

7 Wang W Z, Guo X, Duan C, et al. Comparative analysis of gene expression profiles between the normal human cartilage and the one with endemic osteoarthritis. Osteoarthritis Cartilage, 2009, 17: 83-90

8 Shi T, Mazumdar T, Devecchio J, et al. Cdna microarray gene expression profiling of hedgehog signaling pathway inhibition in human colon cancer cells. PLoS One, 2010, 5: e13054

9 Mehta R G, Whyte L, Huang Y Y, et al. Molecular mechanisms of resveratrol action in lung cancer cells using dual protein and microarray analyses. Cancer Res, 2007, 67: 12007-12017

10 Andrews J, Kennette W, Pilon J, et al. Multi-platform whole-genome microarray analyses refine the epigenetic signature of breast cancer metastasis with gene expression and copy number. PLoS One, 2010, 5: e8665

11 Lee H M, Sugino H, Aoki C, et al. Abnormal networks of immune response-related molecules in bone marrow cells from patients with rheumatoid arthritis as revealed by DNA microarray analysis. Arthritis Res Ther, 2011, 13: R89

12 Bai $\mathrm{X}$, Wu J, Zhang $\mathrm{Q}$, et al. Third-generation human mitochondria-focused cdna microarray and its bioinformatic tools for analysis of gene expression. Biotechniques, 2007, 42: 365-375

13 Green D R, Reed J C. Mitochondria and apoptosis. Science, 1998,
281: 1309-1312

14 Chittenden T, Flemington C, Houghton A B, et al. A conserved domain in bak, distinct from bh1 and bh2, mediates cell death and protein binding functions. EMBO J, 1995, 14: 5589-5596

15 Jurgensmeier J M, Xie Z, Deveraux Q, et al. Bax directly induces release of cytochrome $\mathrm{c}$ from isolated mitochondria. Proc Natl Acad Sci USA, 1998, 95: 4997-5002

16 Malladi S, Challa-Malladi M, Fearnhead H O, et al. The apaf1 *procaspase-9 apoptosome complex functions as a proteolytic-based molecular timer. EMBO J, 2009, 28: 1916-1925

17 Zou H, Li Y, Liu X, et al. An apaf-1.Cytochrome c multimeric complex is a functional apoptosome that activates procaspase-9. J Biol Chem, 1999, 274: 11549-11556

18 Robertus J D, Suh J K, Poulsen L L, et al. Yeast flavin-containing monooxygenase generates oxidizing equivalents that control protein folding in the endoplasmic reticulum. Proc Natl Acad Sci USA, 1999, 96: 2687-2691

19 Xiong Y M, Mo X Y, Zou X Z, et al. Association study between polymorphisms in selenoprotein genes and susceptibility to kashin-beck disease. Osteoarthritis Cartilage, 2010, 18: 817-824

20 Sugden M C, Holness M J. Recent advances in mechanisms regulating glucose oxidation at the level of the pyruvate dehydrogenase complex by pdks. Am J Physiol-Endoc M, 2003, 284: E855-E862

21 Hatefi Y. The mitochondrial electron-transport and oxidativephosphorylation system. Annu Rev Biochem, 1985, 54: 1015-1069

22 Butow R A, Epstein C B, Waddle J A, et al. Genome-wide responses to mitochondrial dysfunction. Mol Biol Cell, 2001, 12: 297-308

23 Cortopassi G A, Wong A. Mitochondria in organismal aging and degeneration. Bba-Bioenergetics, 1999, 1410: 183-193

24 Wallace D C. Mitochondrial diseases in man and mouse. Science, 1999, 283: 1482-1488

25 Brenner C, Kroemer G. Apoptosis. Mitochondria - the death signal integrators. Science, 2000, 289: 1150-1151

26 Hawkes W C, Alkan Z. Regulation of redox signaling by selenoproteins. Biol Trace Elem Res, 2010, 134: 235-251

27 Gibson B W. The human mitochondrial proteome: oxidative stress, protein modifications and oxidative phosphorylation. Int J Biochem Cell B, 2005, 37: 927-934

28 Shokolenko I, Venediktova N, Bochkareva A, et al. Oxidative stress induces degradation of mitochondrial DNA. Nucleic Acids Res, 2009, 37: 2539-2548

Open Access This article is distributed under the terms of the Creative Commons Attribution License which permits any use, distribution, and reproduction in any medium, provided the original author(s) and source are credited. 